\title{
A Low Power Sensor Interface IC for Wearable Wireless Biomedical Devices
}

\author{
Xiaodan Zou \\ Dept of ECE \\ National University of \\ Singapore \\ 4 Engineering Drive 3 \\ Singapore 117576 \\ xiaodan@nus.edu.sg
}

\author{
Xiaoyuan Xu \\ Dept of ECE \\ National University of \\ Singapore \\ 4 Engineering Drive 3 \\ Singapore 117576 \\ elexxy@nus.edu.sg
}

\author{
Yong Lian \\ Dept of ECE \\ National University of \\ Singapore \\ 4 Engineering Drive 3 \\ Singapore 117576 \\ eleliany@nus.edu.sg
}

\author{
Yuanjin Zheng \\ Institute of Microelectronics \\ 11 Science Park Road \\ Singapore Science Park II \\ Singapore 117685 \\ yuanjin@ime.a- \\ star.edu.sg
}

\begin{abstract}
This paper presents a low power, low noise CMOS front-end IC for wearable biomedical sensor devices used in wireless body area network. The IC includes a rail-to-rail low noise amplifier (LNA), a unity gain buffer, and a 12-bit analog-to-digital converter (ADC), which is able to acquire biomedical signals in the range of few $\mu \mathrm{V}$ to $10 \mathrm{mV}$. The overall system operates under $1 \mathrm{~V}$ supply voltage, provides $40 \mathrm{~dB}$ midband gain with $2.4 \mu \mathrm{Vrms}$ input referred noise integrated from $0.04 \mathrm{~Hz}$ to $250 \mathrm{~Hz}$, and consumes $1.4 \mu \mathrm{W}$ when implemented in standard $0.35 \mu \mathrm{m}$ CMOS process. The low power is achieved through a newly introduced system architecture that significantly reduces the clock frequency of ADC compared to the design in [4].
\end{abstract}

\section{Keywords}

Biomedical circuits and systems, wireless biomedical device, biosignal acquisition unit.

\section{INTRODUCTION}

The converging of bioengineering, computers, communications, and nanotechnologies opens the door for integration of wireless communication channel, biomedical sensor devices, and signal processing unit into a single chip, which leads to the introduction of wearable wireless biomedical sensor devices. A collection of these wearable wireless biomedical sensor devices forms the base of a wireless body area network (WBAN). The WBAN not only provides health monitoring to hospital patients but also helps maintain the current state of health of the chronically ill patients and prevents their condition from worsening at home through the telemedicine. It is envisaged that the WBAN will revolutionize the hospital, homecare, and personal health systems and establish a prevention-oriented, consumer-driven model for health care.

A typical WBAN node includes one or more biomedical sensors, a low noise amplifier, an analog-to-digital converter, a digital signal processing unit or microcontroller, and a wireless communication channel. The development of WBAN devices is very challenging due to its small form factor and power

Permission to make digital or hard copies of all or part of this work for personal or classroom use is granted without fee provided that copies are not made or distributed for profit or commercial advantage and that copies bear this notice and the full citation on the first page. To copy otherwise, to republish, to post on servers or to redistribute to lists,requires prior specific permission and/or a fee. BODYNETS 2007, June 11-13, Florence, Italy Copyright () 2007 ICST 978-963-06-2193-9

DOI 10.4108/bodynets.2007.94 constrains. The LNA should provide sufficient gain and deliver a reasonable good noise performance, especially in the low frequency ranging from DC to few hundred hertz. The ADC needs sufficient resolution to resolve the weak bio-signal. The signal processing unit must be well balanced between the processing capability and power dissipation. These requirements are difficult to satisfy due to the flicker noise of CMOS transistors in the low frequency band and tight power budget for the overall device. There are few attempts [1-4] that try to fulfill part of functions required by a WBAN device. One of the impressive solutions was proposed in [4] where a biosignal acquisition system contains a LNA and an 11-bit ADC consuming as low as $2.3 \mu \mathrm{W}$. However, the conversion clock frequency used in [4] is around $20 \mathrm{MHz}$ for a $1 \mathrm{KS} / \mathrm{s}$ ADC resulting in relative high power consumption. In this paper, a low power, low noise signal acquisition system is presented, which includes a LNA and a 12bit ADC. The post layout simulation indicates the overall power consumption below $1.4 \mu \mathrm{W}$, a $40 \%$ reduction in power compared to [4].

\section{SYSTEM ARCHITECTURE}

The signal acquisition system in [4] consists of a LNA followed by a successive approximation ADC. The narrowband LNA with less than $250 \mathrm{~Hz}$ in bandwidth has to drive a large capacitor array in ADC resulting in long linear settling time. In order to achieve acceptable tracking error, the authors decrease the hold period to $1 / 500$ of the sampling period. This leads to a high conversion clock frequency of up to $20 \mathrm{MHz}$ for a $1 \mathrm{KS} / \mathrm{s}$ ADC which significantly increases the power consumption of the overall system. In order to reduce the clock frequency, we propose to insert a buffer between the LNA and ADC, which leads to a new system architecture, as shown in Figure 1. The buffer is designed to have a wide bandwidth and high slew rate to fulfill the charge/discharge requirements of the large capacitive load. By employing this architecture, the hold time is extended to almost $4 / 5$ of the sampling period, which significantly reduces the conversion clock frequency. As a result, the power consumption of the comparator and oscillator in the proposed ADC are only $33 \%$ and $40 \%$ of those in [4], i.e. up to $700 \mathrm{nW}$ saving in power.

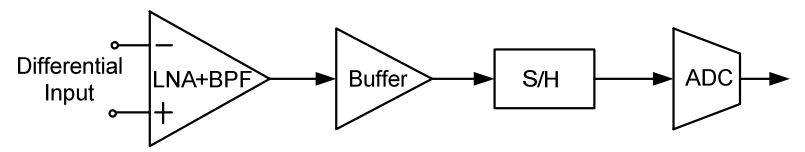

Figure 1. Front-end block diagram 


\section{CIRCUIT DESIGN AND SIMULATION}

The LNA employs the structure proposed in [1]. It employs capacitive feedback and the midband gain is $40 \mathrm{~dB}$ which is determined by the capacitance ratio. The DC rejection is achieved primarily through enhanced pseudoresistors, which eliminate the need for external capacitors, favoring a reasonable input impedance level. The LNA cutoff frequency at the high end is determined by the ratio of the unity gain frequency of the OTA and the midband gain of LNA. The low noise OTA in [4] is adopted as the first stage. Large size NMOS transistors are chosen as the input pair to achieve low input referred noise. The use of NMOS transistors instead of PMOS is a trade-off between supply voltage and noise performance.

A unity gain buffer is implemented after the LNA block in order to fulfill the linear settling time and slew rate requirement. It employs the similar feedback network as in [1] with the capacitance ratio set to 1 . This ensures rail-to-rail input swing for the buffer. The bandwidth of the buffer is designed in the range of $\mathrm{kHz}$. In order to achieve wide bandwidth with considerably low power consumption, an opamp with class $A B$ output stage is chosen to generate a larger output current than the quiescent current [2].

A 12-bit ADC is implemented using the SAR architecture. The sample-and-hold function is realized by the bootstrapped analog switch and capacitor array. The bottom plates of the capacitors are toggled between high and low reference voltages by the SAR logic in order to implement the binary search algorithm, results being reflected on the common node of the array and collected by the comparator.

The overall front-end system has been implemented by standard $0.35 \mu \mathrm{m}$ CMOS technology with Vthp + Vthn $=1.15 \mathrm{~V}$. Figure 2 shows the post-layout simulation of LNA frequency response. The midband gain is $40 \mathrm{~dB}$. The input referred noise is $2.4 \mu \mathrm{Vrms}$ integrated from $0.04 \mathrm{~Hz}$ to $250 \mathrm{~Hz}$, as shown in Figure 3 . A comparison of proposed front-end IC and [4] is summarized in Table 1.

\section{CONCLUSIONS}

We have presented a signal acquisition IC for wearable biomedical devices. It consists of a low power, low noise amplifier with bandpass function, a wide bandwidth and high slew rate buffer, and a 12-bit ADC. The insertion of the buffer greatly improves the system performance and plays a critical role in reducing power. The overall system consumes $1.4 \mu \mathrm{W}$ under $1 \mathrm{~V}$ supply voltage and achieves $40 \mathrm{~dB}$ midband gain with $2.4 \mu \mathrm{Vrms}$ input referred noise.

\section{ACKNOWLEDGEMENT}

This work was supported by Singapore Agency for Science Technology and Research (A*STAR) under Thematic Strategic Research Programme: Embedded \& Hybrid System II.

\section{REFERENCES}

[1] Harrison, R.R. and Charles. C. A Low-Power Low Noise CMOS Amplifier for Neural Recording Applications. IEEE Journal of Solid-State Circuits, 38, 6, (June 2003), 958-965.
[2] Lopez-Morillo, E. Carvajal, R. G. Galan, J. RodriguesVillegas, E. Lopez-Martin, A. and Ramirez-Angulo, J., A Low-Voltage Low-Power QFG-based Sigma-Delta Modulator for Electroencephalogram Applications. In Proceedings of the 2006 IEEE International Conference on Biomedical Circuits and Systems (BioCAS06), (London, UK, Nov. 29 - Dec. 1 2006). 2006.

[3] Ng, K. A. and Chan, P. K. A CMOS Analog Front-End IC for Portable EEG/ECG Monitoring Applications. IEEE Transactions on Circuits and Systems-I, 52, 11, (Nov. 2005), 2335-2346.

[4] $\mathrm{Wu}, \mathrm{H}$. and $\mathrm{Xu}, \mathrm{Y} . \mathrm{P} . \mathrm{A} 1 \mathrm{~V} 2.3 \mu \mathrm{W}$ Biomedical Signal Acquisition IC. In Proceedings of the 2006 IEEE International Solid-State Circuit Conference (ISSCC2006), (San Francisco, USA, Feb. 5-9, 2006). 2006, 119-128.

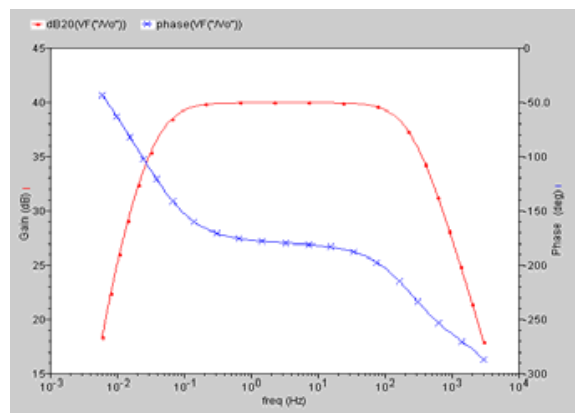

Figure 2. Frequency response of LNA

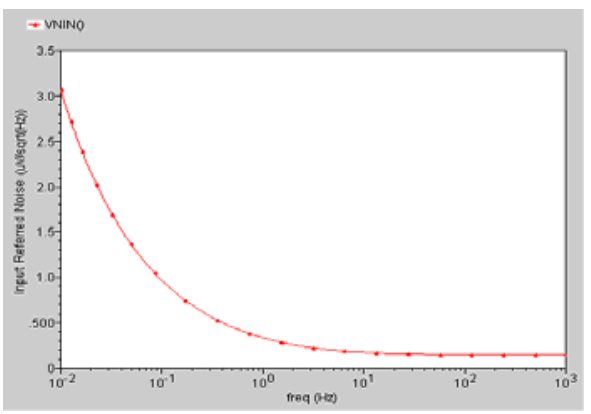

Figure 3. Input referred noise of LNA

Table 1. Comparison of the frond-end IC

\begin{tabular}{|c|c|c|}
\hline Parameter & $\begin{array}{c}\text { Design in [4] } \\
\text { Measured }\end{array}$ & $\begin{array}{c}\text { Proposed } \\
\text { Simulated }\end{array}$ \\
\hline Supply voltage & $1 \mathrm{~V}$ & $1 \mathrm{~V}$ \\
\hline Current (LNA) & $330 \mathrm{nA}$ & $320 \mathrm{nA}$ \\
\hline Midband gain & $40.2 \mathrm{~dB}$ & $40 \mathrm{~dB}$ \\
\hline 3dB Bandwidth & $0.003 \mathrm{~Hz} / 245 \mathrm{~Hz}$ & $0.04 \mathrm{~Hz} / 250 \mathrm{~Hz}$ \\
\hline Input referred noise & $\begin{array}{c}2.7 \mu \mathrm{Vms} \\
\text { (integrated from } \\
0.05 \mathrm{~Hz} \text { to } 245 \mathrm{~Hz})\end{array}$ & $\begin{array}{c}2.4 \mu \mathrm{Vms} \\
\text { (integrated from } \\
0.04 \mathrm{~Hz} \text { to } 250 \mathrm{~Hz})\end{array}$ \\
\hline LNA THD & $0.053 \%$ & $0.023 \%$ \\
\hline CMRR & $>60 \mathrm{~dB}(1 \sim 250 \mathrm{~Hz})$ & $>80 \mathrm{~dB}(0 \sim 250 \mathrm{~Hz})$ \\
\hline ADC resolution & $11-\mathrm{bit}$ & $12-\mathrm{bit}$ \\
\hline ADC sampling rate & $1 \mathrm{KS} / \mathrm{s}$ & $1 \mathrm{KS} / \mathrm{s}$ \\
\hline Total current & $2.3 \mu \mathrm{A}$ & $1.37 \mu \mathrm{A}$ \\
\hline
\end{tabular}

